Received: Apr 23, 2019; Accepted: May 14, 2019; Published: Jun 17, 2019; Paper Id.: IJMPERDAUG201912

\title{
INTRODUCTION
}

The study of Non-Newtonian fluids has a vital role in fields like food processing, polymer processing, etc. Hayat et al. [1] Nadeem and Saleem[2], Tasawar Hayat et al. [3], Macharla Jayachandra Babu et al. [4], Vijayalakshmi and Shankar Bandari. [5], Asha and Sunitha[6], Mallick and Misra [7] and Misra and Sinha [8] are some of the researchers who have focused on MHD Eyring-Powell Nanofluid in recent years. A few recent investigators that may be mentioned are Motsa and Shateyi[9], Shehzad et al.[10], Rizwanulhaq et al. [11], Mustafa and Junaid Ahmad Khan [12], Imran Ullah et al. [13], Sreekala and Kesavareddy [14], Kamran et al. [15], Rajib Biswas and Sardar Firoz Ahmmed [16], Pudhari srilatha[17], Jannath Begam et al.[18], Deivanayaki et al.[19], Jannath Begam et al. [20]and Jannath Begam et al. [21].

Here, we attempt to examine the hall current and ion slip effects on pulsatile flow of some NonNewtonian fluids with thermal radiation.

\section{MATHEMATICAL FORMULATION}

The constitutive equation for Eyring-Powell nanofluid and Casson nanofluid is

$$
\tau_{\mathrm{ij}}=\mu_{\mathrm{nf}} \frac{\partial \mathrm{V}_{\mathrm{i}}}{\partial \mathrm{x}_{\mathrm{j}}}+\frac{1}{\beta} \sin \mathrm{h}^{-1}\left(\frac{1}{\mathrm{c}} \frac{\partial \mathrm{v}_{\mathrm{i}}}{\partial \mathrm{x}_{\mathrm{j}}}\right)
$$


Since $\sin \mathrm{h}^{-1} \mathrm{x} \approx \mathrm{x},|\mathrm{x}|<<1$, then

$$
\begin{aligned}
& \tau_{\mathrm{ij}}=\mu_{\mathrm{nf}} \frac{\partial \mathrm{V}_{\mathrm{i}}}{\partial \mathrm{x}_{\mathrm{j}}}+\frac{1}{\beta}\left(\frac{1}{\mathrm{c}} \frac{\partial \mathrm{V}_{\mathrm{i}}}{\partial \mathrm{x}_{\mathrm{j}}}\right) \\
& =\mu_{\mathrm{nf}}\left(1+\frac{1}{\beta c \mu_{\mathrm{nf}}}\right) \frac{\partial \mathrm{v}_{\mathrm{i}}}{\partial \mathrm{x}_{\mathrm{j}}} \\
& \tau_{\mathrm{ij}}=\left\{\begin{array}{l}
2\left(\mu \mathrm{B}+\frac{\mathrm{P}_{\mathrm{y}^{*}}}{\sqrt{2 \pi}}\right) \mathrm{e}_{\mathrm{ij}}, \pi>\pi_{\mathrm{c}} \\
2\left(\mu \mathrm{B}+\frac{\mathrm{P}_{\mathrm{y}^{*}}}{\sqrt{2 \pi_{\mathrm{c}}}}\right) \mathrm{e}_{\mathrm{ij}}, \pi<\pi_{\mathrm{c}}
\end{array}\right\} \\
& \tau_{\mathrm{ij}}=\mu_{\mathrm{nf}}\left(1+\frac{1}{\beta}\right) \frac{\partial \mathrm{v}_{\mathrm{i}}}{\partial \mathrm{x}_{\mathrm{j}}}
\end{aligned}
$$

Unsteady incompressible Eyring-Powell nanofluid and Casson nanofluid are examined, with its X-axis selected as lower plate and transversely applied magnetic field along y axis. Using Boussinesq approximation, we have

$$
\begin{aligned}
& \frac{\partial \mathrm{u}^{*}}{\partial \mathrm{t}^{*}}=-\frac{1}{\rho_{\mathrm{nf}}} \frac{\partial \mathrm{P}^{*}}{\partial \mathrm{x}^{*}}+\mathrm{q} \vartheta_{\mathrm{nf}}\left(1+\frac{1}{\beta c \mu_{\mathrm{nf}}}\right) \frac{\partial^{2} \mathrm{u}^{*}}{\partial \mathrm{y}^{* 2}}+(1-\mathrm{q}) \mathrm{q} \vartheta_{\mathrm{nf}}\left(1+\frac{1}{\beta}\right) \frac{\partial^{2} \mathrm{u}^{*}}{\partial \mathrm{y}^{* 2}}-\frac{\mu_{\mathrm{nf}}}{\rho_{\mathrm{nf}}} \mathrm{u}^{*}+\frac{\sigma_{\mathrm{nf}} \mathrm{B}_{0}^{2}\left(1+\beta_{\mathrm{e}} \beta_{\mathrm{i}}\right)}{\rho_{\mathrm{nf}}\left[\left(1+\beta_{\mathrm{e}} \beta_{\mathrm{i}}\right)^{2}+\beta_{\mathrm{e}}^{2}\right]} \mathrm{u}^{*} \\
& \frac{\partial \mathrm{T}^{*}}{\partial \mathrm{t}^{*}}=\frac{\mathrm{k}_{\mathrm{nf}}}{\left(\rho \mathrm{C}_{\mathrm{P}}\right)_{\mathrm{nf}}} \frac{\partial^{2} \mathrm{~T}^{*}}{\partial \mathrm{y}^{* 2}}+\frac{1}{\left(\rho \mathrm{C}_{\mathrm{P}}\right)_{\mathrm{nf}}} \frac{\partial \mathrm{q}_{\mathrm{r}}}{\partial \mathrm{y}^{*}}+\mathrm{q} \frac{\mu_{\mathrm{nf}}\left(1+\frac{1}{\beta c \mu_{\mathrm{nf}}}\right)}{\left(\rho \mathrm{C}_{\mathrm{P}}\right)_{\mathrm{nf}}}\left(\frac{\partial \mathrm{u}^{*}}{\partial \mathrm{y}^{*}}\right)^{2}+(1-\mathrm{q}) \frac{\mu_{\mathrm{nf}}\left(1+\frac{1}{\beta}\right)}{\left(\rho \mathrm{C}_{\mathrm{P}}\right)_{\mathrm{nf}}}\left(\frac{\partial \mathrm{u}^{*}}{\partial \mathrm{y}^{*}}\right)^{2}+\frac{\mathrm{Q}_{0}}{\left(\rho \mathrm{C}_{\mathrm{P}}\right)_{\mathrm{nf}}}\left(\mathrm{T}^{*}-\mathrm{T}_{0}\right)
\end{aligned}
$$

In equations (4) and (5), $\mathrm{q} \epsilon[0,1]$, it states Eyring-Powell nanofluid for $\mathrm{q}=1$ and Casson nanofluid for $\mathrm{q}=0$.

and

$$
\begin{aligned}
& u^{*}=0, \mathrm{~T}^{*}=T_{0} \text { aty } y^{*}=0 \\
& u^{*}=0, \mathrm{~T}^{*}=T_{1} \text { aty } y^{*}=h
\end{aligned}
$$

The physical properties of nanofluid such as $\mu_{n f}, \rho_{n f},\left(\rho C_{P}\right)_{n f}$ and $k_{n f}$ are given as

$$
\begin{aligned}
& \mu_{n f}=\frac{\mu_{f}}{(1-\phi)^{2.5}} \\
& \rho_{n f}=(1-\phi) \rho_{f}+\phi \rho_{s} \\
& \left(\rho C_{P}\right)_{n f}=(1-\phi)\left(\rho C_{P}\right)_{f}+\phi\left(\rho C_{P}\right)_{s} \\
& \frac{k_{n f}}{k_{f}}=\frac{k_{s}+2 k_{f}-2 \phi\left(k_{f}-k_{S}\right)}{k_{s}+2 k_{f}+\phi\left(k_{f}-k_{s}\right)} \\
& q_{r}=-\frac{4 \sigma^{*}}{3 \mathrm{k}^{*}} \frac{\partial \mathrm{T}^{* 4}}{\partial \mathrm{y}^{*}} \\
& \mathrm{~T}^{* 4} \cong 4 T_{1}^{3} T^{*}-3 T_{1}^{4}
\end{aligned}
$$

Using Equations(12) and (13), Equation (5) becomes

$$
\begin{aligned}
& \frac{\partial \mathrm{T}^{*}}{\partial \mathrm{t}^{*}}=\frac{\mathrm{k}_{\mathrm{nf}}}{\left(\rho \mathrm{C}_{\mathrm{P}}\right)_{\mathrm{nf}}} \frac{\partial^{2} \mathrm{~T}^{*}}{\partial \mathrm{y}^{* 2}}+\frac{1}{\left(\rho \mathrm{C}_{\mathrm{P}}\right)_{\mathrm{nf}}}\left[\frac{16 \sigma^{*}}{3 \mathrm{k}^{*}} \mathrm{~T}_{1}^{3} \frac{\partial^{2} \mathrm{~T}^{*}}{\partial \mathrm{y}^{* 2}}\right]+\mathrm{q} \frac{\mu_{\mathrm{nf}}\left(1+\frac{1}{\beta c \mu_{\mathrm{nf}}}\right)}{\left(\rho \mathrm{C}_{\mathrm{P}}\right)_{\mathrm{nf}}}\left(\frac{\partial \mathrm{u}^{*}}{\partial \mathrm{y}^{*}}\right)^{2}+(1-\mathrm{q}) \frac{\mu_{\mathrm{nf}}\left(1+\frac{1}{\beta}\right)}{\left(\rho \mathrm{C}_{\mathrm{P}}\right)_{\mathrm{nf}}}\left(\frac{\partial \mathrm{u}^{*}}{\partial \mathrm{y}^{*}}\right)^{2}+\frac{\mathrm{Q}_{0}}{\left(\rho \mathrm{C}_{\mathrm{P}}\right)_{\mathrm{nf}}}\left(\mathrm{T}^{*}-\mathrm{T}_{0}\right) \\
& -\frac{1}{\rho_{f}} \frac{\partial \mathrm{P}^{*}}{\partial \mathrm{x}^{*}}=A\left(1+\epsilon e^{i w t}\right)
\end{aligned}
$$


Introducing the following dimensionless variables

$u=\frac{u^{*} \omega}{A}, t=t^{*} \omega, x=\frac{x^{*}}{h}, y=\frac{y^{*}}{h}, \theta=\frac{T^{*}-T_{0}}{T_{1}-T_{0}}, P=\frac{P^{*}}{A_{\rho_{f}} h^{\prime}}, k_{0}=\frac{1}{\beta c \mu_{f}}$

And parameters as in Equations (8) to (11) into Equations (15),(4) and (14), we get

$-\frac{\partial \mathrm{P}}{\partial \mathrm{x}}=1+\epsilon e^{i t}$

$\frac{\partial \mathrm{u}}{\partial \mathrm{t}}=-\frac{1}{A_{1}} \frac{\partial \mathrm{P}}{\partial \mathrm{x}}+q \frac{A_{2}}{A_{1}}\left(1+\frac{k_{0}}{A_{2}}\right) \frac{1}{R} \frac{\partial^{2} u}{\partial \mathrm{y}^{2}}+(1-q) \frac{A_{2}}{A_{1}}\left(1+\frac{1}{\beta}\right) \frac{1}{R} \frac{\partial^{2} u}{\partial \mathrm{y}^{2}}-\frac{A_{2}}{A_{1}}\left(\frac{1}{R D_{a}}+M_{1}\right) u$

$\frac{\partial \theta}{\partial \mathrm{t}}=\left(\frac{A_{4}}{A_{3}}+\frac{4 R d}{3 A_{3}}\right) \frac{1}{R P_{r}} \frac{\partial^{2} \theta}{\partial \mathrm{y}^{2}}+q \frac{A_{2}}{A_{3}}\left(1+\frac{k_{0}}{A_{2}}\right) \frac{E c}{R}\left(\frac{\partial \mathrm{u}}{\partial \mathrm{y}}\right)^{2}+(1-q) \frac{A_{2}}{A_{3}}\left(1+\frac{1}{\beta}\right) \frac{E c}{R}\left(\frac{\partial \mathrm{u}}{\partial \mathrm{y}}\right)^{2}+\frac{Q}{A_{3} R} \theta$

and

$u=0, \theta=0$ aty $=0$

$u=0, \theta=1$ aty $=1$

where $A_{1}=(1-\phi)+\phi \frac{\rho_{s}}{\rho_{f}}, A_{2}=\frac{1}{(1-\phi)^{2.5}}$,

$A_{3}=(1-\phi)+\phi \frac{\left(\rho C_{P}\right)_{s}}{\left(\rho C_{P}\right)_{f}}, A_{4}=\frac{k_{s}+2 k_{f}-2 \phi\left(k_{f}-k_{S}\right)}{k_{s}+2 k_{f}+\phi\left(k_{f}-k_{S}\right)}$,

$\operatorname{Pr}=\frac{\left(\rho C_{P}\right)_{f v f}}{k_{f}}, \mathrm{Ec}=\frac{A^{2}}{\omega^{2}\left(\rho C_{P}\right)_{f}\left(T_{1}-T_{0}\right)}, \mathrm{R}=\frac{\omega h^{2}}{\mu_{f}}, \mathrm{k}_{0}=\frac{1}{\beta c \mu_{f}}, \mathrm{M}=\frac{\sigma_{n f} B_{0}^{2}}{\rho_{n f}}$

$\mathrm{Da}=\frac{k}{h^{2}}, \mathrm{Rd}=\frac{4 \sigma^{*} T_{1}^{3}}{k_{f} k^{*}}, \mathrm{Q}=\frac{Q_{0} h^{2}}{\left(\rho C_{P}\right)_{f} \mu_{f}}, \mathrm{M}_{1}=\frac{M\left(1+\beta_{e} \beta_{i}\right)}{\left(1+\beta_{e} \beta_{i}\right)^{2}+\beta_{e}^{2}}$

\section{SOLUTION FOR THE PROBLEM}

The $\mathrm{u}$ and $\theta$ are written as

$u=u_{0}(y)+\epsilon u_{1}(y) e^{i t}$

$\theta=\theta_{0}(y)+\epsilon \theta_{1}(y) e^{i t}+\epsilon^{2} \theta_{2}(y) e^{2 i t}$

Using Equations (17), (22) and (23) in Equations (18) and (19) and comparing the terms of like powers of $\epsilon$, we get

$$
\begin{aligned}
& \mathrm{q} \frac{\mathrm{A}_{2}}{\mathrm{~A}_{1}}\left(1+\frac{\mathrm{k}_{0}}{\mathrm{~A}_{2}}\right) \frac{1}{\mathrm{R}} \mathrm{u}_{0}^{\prime \prime}+(1-\mathrm{q}) \frac{\mathrm{A}_{2}}{\mathrm{~A}_{1}}\left(1+\frac{1}{\beta}\right) \frac{1}{\mathrm{R}} \mathrm{u}_{0}^{\prime \prime}-\frac{\mathrm{A}_{2}}{\mathrm{~A}_{1}}\left(\frac{1}{\mathrm{RD}_{\mathrm{a}}}+\mathrm{M}_{1}\right) \mathrm{u}_{0}=-\frac{1}{\mathrm{~A}_{1}} \\
& \mathrm{q} \frac{\mathrm{A}_{2}}{\mathrm{~A}_{1}}\left(1+\frac{\mathrm{k}_{0}}{\mathrm{~A}_{2}}\right) \frac{1}{\mathrm{R}} \mathrm{u}_{1}^{\prime \prime}+(1-\mathrm{q}) \frac{\mathrm{A}_{2}}{\mathrm{~A}_{1}}\left(1+\frac{1}{\beta}\right) \frac{1}{\mathrm{R}} \mathrm{u}_{1}^{\prime \prime}-\frac{\mathrm{A}_{2}}{\mathrm{~A}_{1}}\left(\frac{1}{\mathrm{RD}_{\mathrm{a}}}+\mathrm{M}_{1}\right) \mathrm{u}_{1}-i \mathrm{u}_{1}=-\frac{1}{\mathrm{~A}_{1}} \\
& \left(\frac{\mathrm{A}_{4}}{\mathrm{~A}_{3}}+\frac{4 \mathrm{Rd}}{3 \mathrm{~A}_{3}}\right) \frac{1}{\mathrm{RP} \mathrm{r}} \theta_{0}^{\prime \prime}+\mathrm{q} \frac{\mathrm{A}_{2}}{\mathrm{~A}_{3}}\left(1+\frac{\mathrm{k}_{0}}{\mathrm{~A}_{2}}\right)\left(\mathrm{u}_{0}^{\prime}\right)^{2}+(1-\mathrm{q}) \frac{\mathrm{A}_{2}}{\mathrm{~A}_{3}}\left(1+\frac{1}{\beta}\right)\left(\mathrm{u}_{0}^{\prime}\right)^{2}+\frac{\mathrm{Q}}{\mathrm{A}_{3} \mathrm{R}} \theta_{0}=0 \\
& \left(\frac{\mathrm{A}_{4}}{\mathrm{~A}_{3}}+\frac{4 \mathrm{Rd}}{3 \mathrm{~A}_{3}}\right) \frac{1}{\mathrm{RP}_{\mathrm{r}}} \theta_{1}^{\prime \prime}+2 \mathrm{q} \frac{\mathrm{A}_{2}}{\mathrm{~A}_{3}}\left(1+\frac{\mathrm{k}_{0}}{\mathrm{~A}_{2}}\right) \mathrm{u}_{0}^{\prime} \mathrm{u}_{1}^{\prime}+2(1-\mathrm{q}) \frac{\mathrm{A}_{2}}{\mathrm{~A}_{3}}\left(1+\frac{1}{\beta}\right) \mathrm{u}_{0}^{\prime} \mathrm{u}_{1}^{\prime} \frac{\mathrm{Q}}{\mathrm{A}_{3} \mathrm{R}} \theta_{1}-i \theta_{1}=0 \\
& \left(\frac{\mathrm{A}_{4}}{\mathrm{~A}_{3}}+\frac{4 \mathrm{Rd}}{3 \mathrm{~A}_{3}}\right) \frac{1}{\mathrm{RP}} \theta_{2}^{\prime \prime}+2 \mathrm{q} \frac{\mathrm{A}_{2}}{\mathrm{~A}_{3}}\left(1+\frac{\mathrm{k}_{0}}{\mathrm{~A}_{2}}\right)\left(\mathrm{u}_{1}^{\prime}\right)^{2}+2(1-\mathrm{q}) \frac{\mathrm{A}_{2}}{\mathrm{~A}_{3}}\left(1+\frac{1}{\beta}\right)\left(\mathrm{u}_{1}^{\prime}\right)^{2}+\frac{\mathrm{Q}}{\mathrm{A}_{3} \mathrm{R}} \theta_{2}-2 i \theta_{2}=0
\end{aligned}
$$

and 


$$
\begin{aligned}
& \mathrm{u}_{0}=0, \theta_{0}=0 \text { at } \mathrm{y}=0 \\
& =0, \theta_{0}=1 \text { at } \mathrm{y}=1 \\
& \mathrm{u}_{1}=0, \theta_{1}=0, \theta_{2}=0 \text { at } \mathrm{y}=0 \\
& u_{1}=0, \theta_{1}=0, \theta_{2}=0 \text { at } y=1
\end{aligned}
$$

By solving equations (24) to (28) with the corresponding boundary conditions (29) to (32), we obtain

$$
\begin{aligned}
& u_{0}=B_{7} e^{\sqrt{B_{3 y}}}+B_{6} e^{-\sqrt{B_{3 y}}}-\frac{B_{4}}{B_{3}} \\
& \mathrm{u}_{1}=\mathrm{B}_{9} \mathrm{e}^{\sqrt{\mathrm{B}_{5 y}}}+\mathrm{B}_{8} \mathrm{e}^{-\sqrt{B_{5 y}}}-\frac{\mathrm{B}_{4}}{\mathrm{~B}_{5}} \\
& \theta_{0}=c_{11} \cos \sqrt{d_{2}} y+c_{12} \sin \sqrt{d_{2}} y+c_{8} e^{2 \sqrt{B_{3} y}}+c_{9} e^{-2 \sqrt{ } B_{3 y}}+c_{10} \\
& \theta_{1}=D_{6} \mathrm{e}^{\sqrt{C_{4} y}}+D_{5} e^{-\sqrt{ } C_{4 y}}+D_{1} e^{m_{1} y}+D_{2} e^{m_{2} y}+D_{3} e^{m_{3} y}+D_{4} e^{m_{4} y} \\
& \theta_{2}=D_{11} e^{\sqrt{ } C_{6 y}}+D_{10} e^{-\sqrt{ } C_{6 y}}+D_{7} e^{2 \sqrt{ } B_{5 y}}+D_{8} e^{-2 \sqrt{ } B_{5 y}}+D_{9}
\end{aligned}
$$

\section{RESULTS AND DISCUSSIONS}

To find the physical insight, the velocity and temperature profiles are studied for different parameters and discussed graphically. Figure (1a) pictures, velocity diminishes for $\mathrm{k}_{0}$ and the opposite behavior has keenly been observed from figure (1b) for the Casson nanofluid parameter for different $\beta_{e}$. Velocity diminishes for ion slip parameter $\beta_{i}$ as illustrated in figure(2a). The contrasting effect has been pointed out in figure (2b). Velocity diminishes as $\mathrm{k}_{0}$ and $\beta$ increase as examined in figure (3a) and (3b). Casson nanofluid shows little high increase in unsteady temperature for various Ec than $\mathrm{k}_{0}$ as analysed in figure(4). $\beta$ shows slightly increasing $\theta_{t}$ than $\mathrm{k}_{0}$ for various heat source parameter $\mathrm{Q}$ is observed from Figure (5).

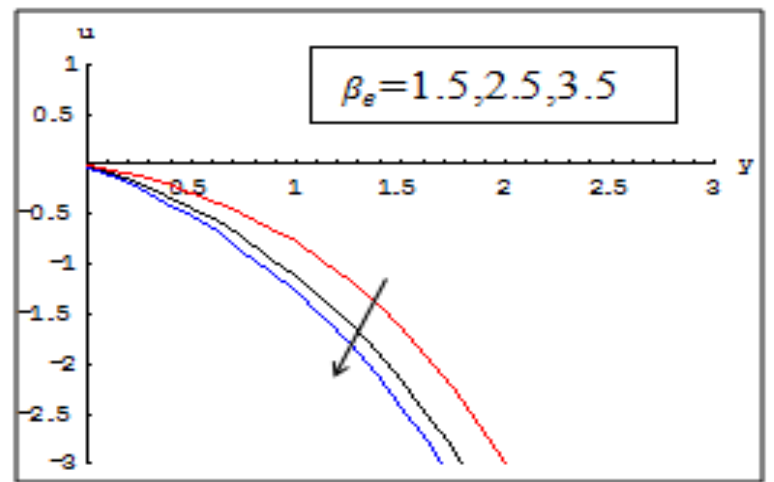

Figure (1a): Impact of $\beta_{e}$ on Velocity Profile $u$ of of Eyring-Powell Nanofluid

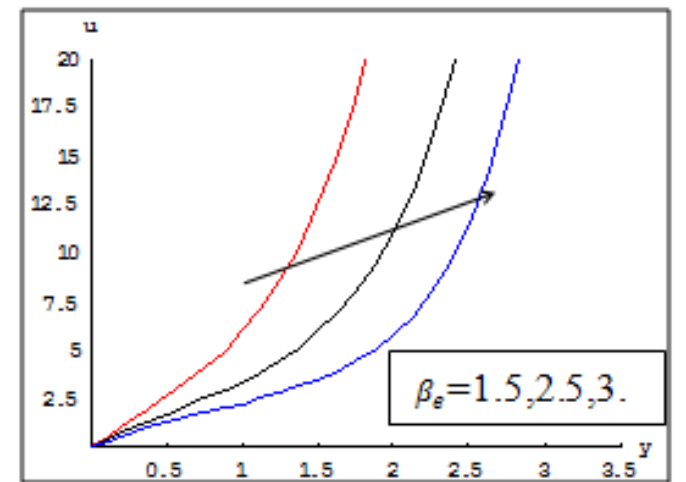

Figure (1b): Impact of $\beta_{e}$ on Velocity Profile u Cass on Nanofluid 


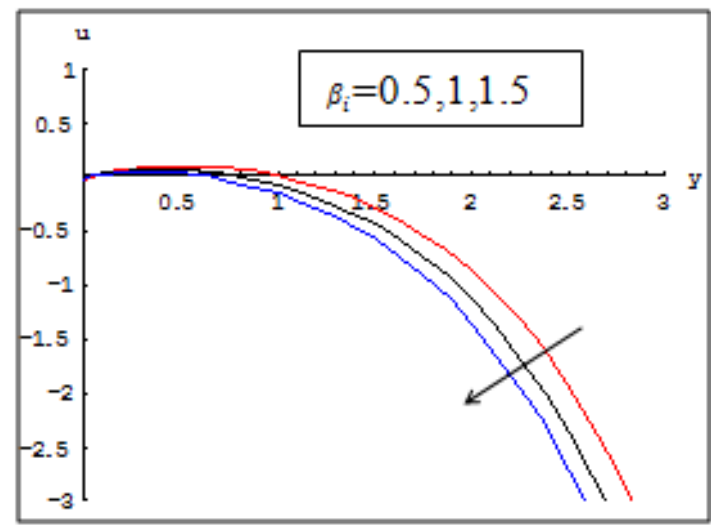

Figure (2a): Impact of $\boldsymbol{\beta}_{i}$ on Velocity Profile u of Eyring-Powell Nanofluid

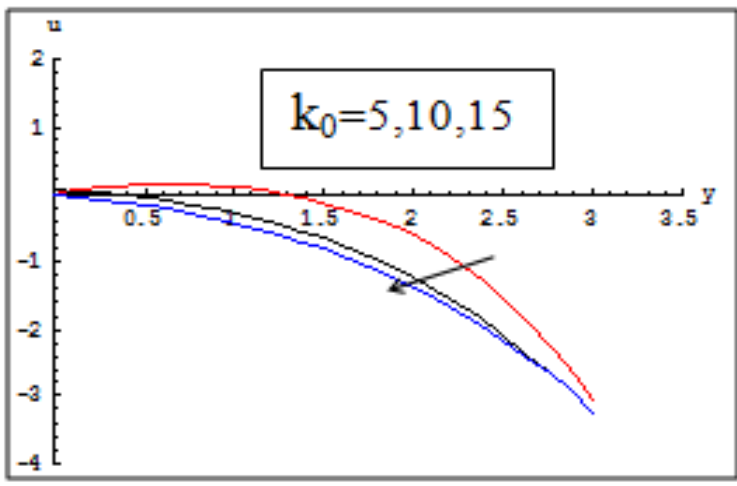

Figure (3a): Impact of $k_{0}$ on Velocity Profile u of Eyring-Powell Nanofluid

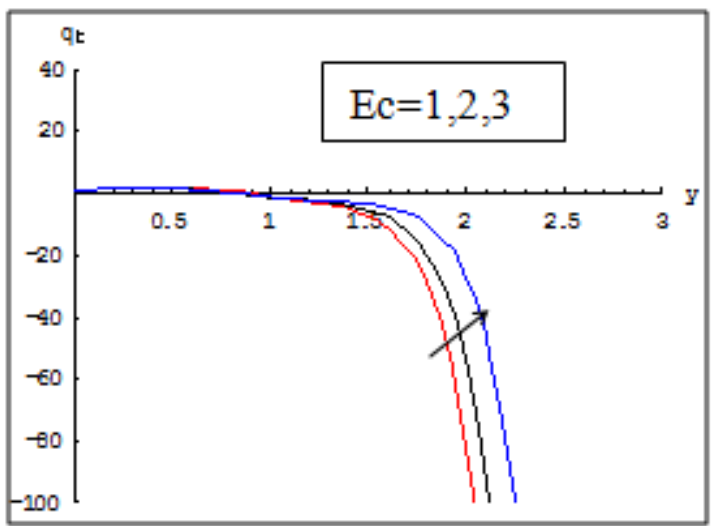

Figure (4a): Impact of Ec on Unsteady Temperature Profile $\theta_{t}$ of Eyring-Powell Nanofluid

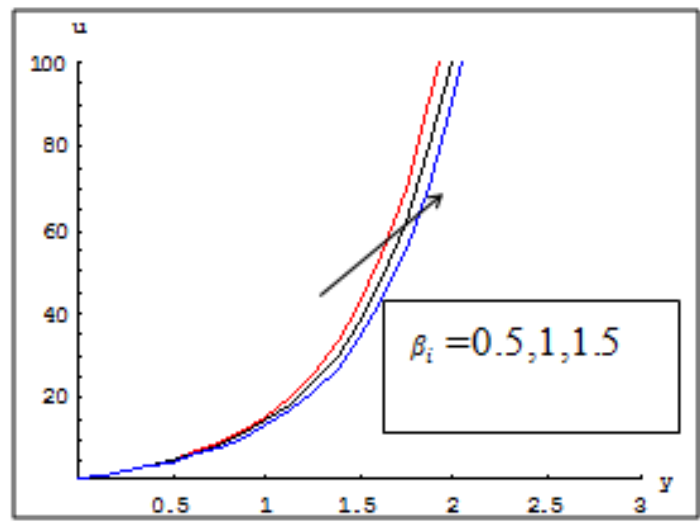

Figure (2b): Impact of $\boldsymbol{\beta}_{i}$ on Velocity Profile u of Cass on Nanofluid

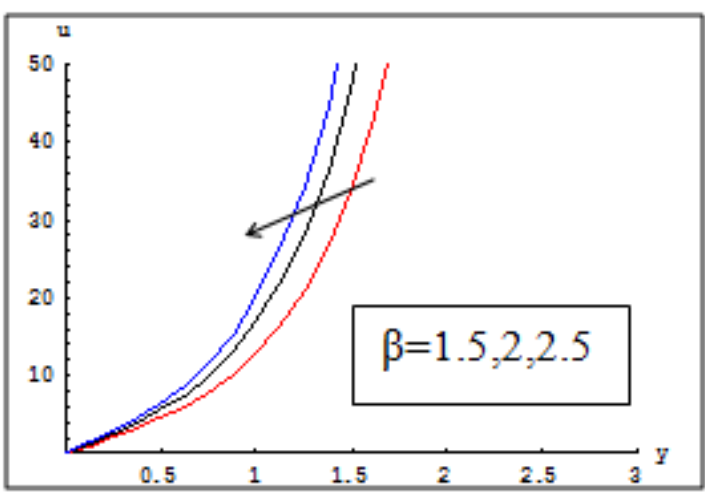

Figure (3b): Impact of $\beta$ on Velocity Profile u of Cass on Nanofluid

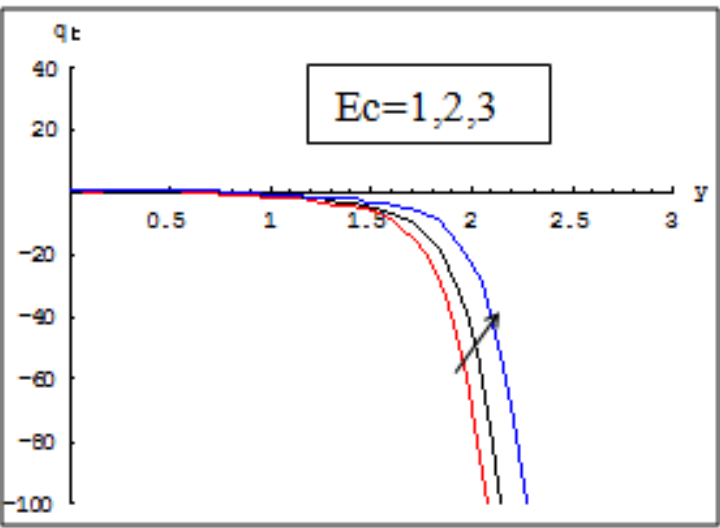

Figure (4b): Impact of Ec on Unsteady Temperature Profile $\theta_{t}$ of Cass on Nanofluid 


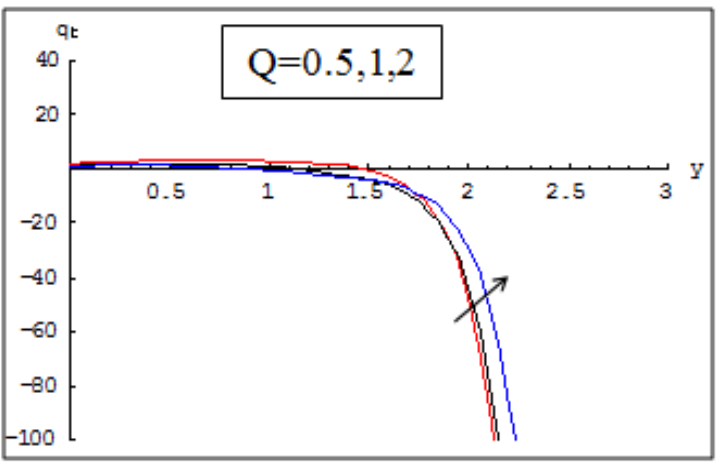

Figure (5a): Impact of $Q$ on Unsteady Temperature Profile $\theta_{t}$ of Eyring-Powell Nanofluid

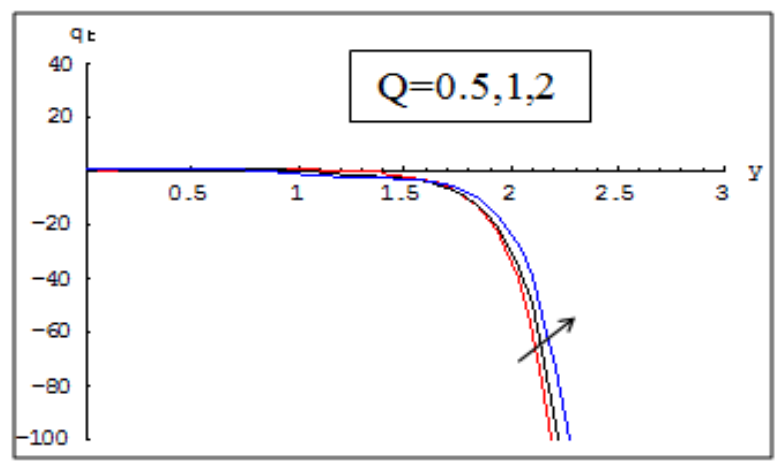

Figure (5b): Impact of $Q$ on Unsteady Temperature Profile $\theta_{t}$ of Cass on Nanofluid

\section{CONCLUSIONS}

Finally, we conclude that,

- $\quad$ The velocity profile decreases with Hall current and ion slip effect for $\mathrm{k}_{0}$ and increase for $\beta$.

- The velocity profile gets decreased with increasing values of $\mathrm{k}_{0}$ and $\beta$, and the temperature profile gets increased with enhanced Ec and $\mathrm{Q}$ for both $\mathrm{k}_{0}$ and $\beta$.

Although a very slight difference is observed between $\mathrm{k}_{0}$ and $\beta$, we conclude that $\mathrm{k}_{0}$ shows minute variations when compared with $\beta$.

\section{REFERENCES}

1. Hayat T, Farooq M, Alsaedi A, Iqbal Z.(2013).Melting heat transfer in the stagnation point flow of Powell-Eyringfluid. Journal of Thermophysics and Heat transfer.27(4):761-766.

2. Nadeem S,Saleem S.(2014).Series solution of unsteady Eyring-Powell nanofluid flow on a rotating cone. Indian Journal of Pure and Applied Physics.52(1):725-737.

3. TasawarHayat, BilalAshraf, Sabir Ali Shehzad, ElbazAbouelmoad. (2015). Three dimensional flow of Eyring-Powell nanofluid over an exponentially stretching sheet. International journal of Numerical methods for Heat and Fluidflow.25(3):593-616.

4. Qashqaei, A., \& ASL, R. G. (2015). Numerical Modeling And Simulation Of Copper Oxide Nanofluids Used In Compact Heat Exchangers. International Journal of Mechanical Engineering, 4 (2), 1, 8.

5. Macharla Jayachandra Babu, Naramgari Sandeep, Chakravarthula SK Raju. (2016). Heat and mass transfer in MHD EyringPowell Nanofluid flow due to cone in porous medium. International Journal of Engineering Research in Africa.19(1):57-74.

6. Vijayalakshmi T, Shankar Bandari. (2017). Stagnation point flow of MHD Eyring-Powell Nanofluid over exponential stretching sheet with convective heat transfer. Journal of Nanofluids. 6(3): 447-456.

7. Asha S.K, Sunitha G. (2016). Mixed convection Peristaltic flow of aEyring-Powell Nanofluid with magnetic field in a nonuniform channel. Journal of applied Mathematics and Computation.2(8):332-344.

8. Hameed, A. A., Al-Fatlawy, N. M., \& Al-Salehi, A. M. (2017). Estimation of hourly global solar radiation incident of inclined surface in Iraq at different sky condition. Int. J. Res. Appl. Nat. Soc. Sci, 5, 13-28.

9. Mallick B, Misra JC.(2019).Peristaltic flow of Eyring-Powell Nanofluid under the action of an electro magnetic field. Engineering science and technology, an International journal. 22(1):266-281. 


\section{Nanofluids with Hall Current and Ion Slip}

\section{Through a Porous Medium}

10. Misra JC, Sinha A. (2013). Effect of thermal radiation on MHD flow of blood and heat transfer in a permeable capillary in stretching motion. Heat and Mass Transfer.49(5):617-628.

11. Motsa SS, Shateyi S. (2012). The effects of chemical reaction, Hall and ion slip currents on MHD Micropolar fluid flow with thermal diffusivity using a novel numerical technique .Journal of applied Mathematics.1(1):1-30.

12. Shehzad SA, Hayat T, Qasim M, Asghar S. (2013). Effects of mass transfer on MHD flow of casson fluid with chemical reaction and suction. Brazelian Journal of Chemical Engineering.30(1):1-10.

13. Rizwanulhaq, Sohail Nadeem, Zafar Hayyat Khan, Toyin Gideon Okeedayo. (2014). Convective heat transfer and MHD effects on Casson nanofluid flow over a shrinking sheet. Central European Journal of Physics.12(12): 862-871.

14. Mustafa M, Junaid Ahmad Khan. (2015). Model for flow of Casson nanofluid past a non-linearly stretching sheet considering magnetic field effects. AIP Advances.5(7):1-20.

15. Imran Ullah, IiyasKhan, SharidanShafie. (2016). MHD natural convection flow of Casson nanofluid over nonlinearly stretching sheet through porous medium with chemical reaction and thermal radiation. Nano scale Research letters.11(1):527537.

16. SreekalaL, Kesavareddy E. (2014). Hall Effects on unsteady MHD flow of a Non-Newtonian fluid through a Porous medium with uniform suction and injection. IOSR Journal of Mechanical and Civil Engineering.11(5):55-64.

17. Kamran A, HussainS, Sageer M, Akmal N. (2017). A numerical study of Magnetohydrodynamics flow in Casson nanofluid combined with joule heating and slip boundary conditions. Results in Physics.7(1): 3037-3048.

18. Rajib Biswas, Sardar Firoz Ahmmed. (2016). Effects of hall current and chemical reaction on Magnetohydrodynamics unsteady heat and mass transfer of Casson nanofluid flow through a vertical plate. Journal of Heat Transfer. 140(9): 1-12.

19. Pudhari Srilatha. (2018). Hall Current Effect on the MHD Flow of Newtonian Fluid through a Porous Medium. International Journal of Applied Engineering Research.13(7): 4637-4651

20. Jannath BegamM, Kowsalya J.(2016).MHD Mass transfer flow past a vertical porous plate embedded in a porous medium in a slip flow regime with the influence of hall current and thermal diffusion. International journal of Advanced scientific and technical research. 5(6):600-613.

21. Krishna, V. M. (2016). Heat Transfer Enhancement by using ZnO Water Nanofluid in a Concentric Tube Heat Exchanger under Forced Convection Conditions. International Journal of Innovations in Engineering and Technology, 7(4), 177-184.

22. Jannath Begam M, Deivanayaki M.(2019). The Pulsatile flow of Eyring-Powell nanofluid with hall effect through a porous medium. Journal of Advanced research in dynamical and control systems.11(4):1400-1406. 


\section{APPENDIX}

\section{NOMENCLATURE}

\begin{tabular}{|l|l|}
\hline$\beta$ & Casson nanofluid parameter \\
\hline$Q_{0}$ & Coefficient of Heat source/Sink \\
\hline $\mathrm{Da}$ & Darcy Number \\
\hline$\rho_{n f}$ & Density of Nanofluid \\
\hline$\rho_{f}$ & Density of the Base fluid \\
\hline$\rho_{s}$ & Density of the Nanoparticle \\
\hline $\mathrm{h}$ & Distance Between the plates \\
\hline$\mu_{n f}$ & Dynamic viscosity of the Nanofluid \\
\hline $\mathrm{Ec}$ & Eckert Number \\
\hline$\left(\rho C_{P}\right)_{n f}$ & Effective Heat Capacitance of Nanofluid \\
\hline $\mathrm{k}_{0}$ & Eyring-Powell nanofluid parameter \\
\hline $\mathrm{R}$ & Frequency parameter \\
\hline$\beta_{e}$ & Hall current parameter \\
\hline$\left(\rho C_{P}\right)_{f}$ & Heat Capacitance of Base fluid \\
\hline$\left(\rho C_{P}\right)_{s}$ & Heat Capacitance of Nanoparticle \\
\hline $\mathrm{Q}$ & Heat Source parameter \\
\hline$\beta_{i}$ & Ion slip parameter \\
\hline $\mathrm{k}^{*}$ & Mean Absorption Coefficient \\
\hline$\phi$ & Nanoparticles volume fraction \\
\hline $\mathrm{k}$ & Permeability of porous medium \\
\hline $\mathrm{Pr}$ & Prandtl number \\
\hline $\mathrm{P} *$ & Pressure \\
\hline $\mathrm{Rd}$ & Radiation Parameter \\
\hline$q_{r}$ & Radiative Heat flux \\
\hline$\sigma^{*}$ & Stefan-Boltzmann Constant \\
\hline$k_{f}$ & Thermal conductivity of Base fluid \\
\hline$k_{n f}$ & Thermal conductivity of Nanofluid \\
\hline$k_{s}$ & Thermal conductivity of Nanoparticle \\
\hline $\mathrm{u} *$ & Velocity component in x*-direction \\
\hline$\mu_{f}$ & Viscosity of the Base fluid \\
\hline & \\
\hline
\end{tabular}

